OPEN ACCESS

Edited by:

Veronica Vella,

University of Catania, Italy

Reviewed by:

Aarti Mathur.

Johns Hopkins Medicine,

United States

Subramanian Kannan,

Narayana Health, India

*Correspondence:

Thera P. Links

t.p.links@umcg.n

${ }^{\dagger}$ These authors have contributed equally to this work and share first authorship

Specialty section: This article was submitted to Cancer Endocrinology, a section of the journal Frontiers in Endocrinology

Received: 19 January 2021 Accepted: 28 April 2021 Published: 28 May 2021

Citation: van der Boom T Zandee WT, Dekkers CCJ, van der Horst-Schrivers ANA, Jansen L, Kruijff S, Brouwers AH and Links TP (2021) The Value of Pre-

Ablative I-131 Scan for Clinical

Management in Patients With Differentiated Thyroid Carcinoma.

Front. Endocrinol. 12:655676. doi: 10.3389/fendo.2021.655676

\section{The Value of Pre-Ablative I-131 Scan for Clinical Management in Patients With Differentiated Thyroid Carcinoma}

Trynke van der Boom ${ }^{1 \dagger}$, Wouter T. Zandee ${ }^{1 \dagger}$, Claire C. J. Dekkers ${ }^{1}$, Anouk N. A. van der Horst-Schrivers ${ }^{1,4}$, Liesbeth Jansen ${ }^{2}$, Schelto Kruijff ${ }^{2}$, Adrienne H. Brouwers ${ }^{3}$ and Thera P. Links ${ }^{1 *}$

${ }^{1}$ Department of Internal Medicine, Division of Endocrinology, University Medical Center Groningen, University of Groningen, Groningen, Netherlands, 2 Department of Surgery, University Medical Center Groningen, University of Groningen, Groningen, Netherlands, ${ }^{3}$ Department of Nuclear Medicine and Molecular Imaging, University Medical Center Groningen, University of Groningen, Groningen, Netherlands, ${ }^{4}$ Department of Emergency Medicine, Maastricht University Medical Center and Maastricht University, Maastricht, Netherlands

Background: A diagnostic I-131 (Dx) scan is used to detect a thyroid remnant or metastases before treatment of differentiated thyroid cancer (DTC) with I-131. The aim of this study is to specify in which patients with DTC a Dx scan could have an additional value, by studying the effect of the Dx scan on clinical management.

Methods: Patients with DTC, treated with I-131 after thyroidectomy were included in this retrospective cohort study. Twenty-four hours after administration of $37 \mathrm{MBq}$ l-131 a whole body Dx scan and an uptake measurement at the original thyroid bed were performed. Outcomes of the Dx scan and the subsequent changes in clinical management, defined as additional surgery or adjustment of I-131 activity, were reported. Risk factors for a change in clinical management were identified with a binary logistic regression.

Results: In 11 (4.2\%) patients clinical management was changed, including additional surgery $(n=5)$, lowering $1-131$ activity $(n=5)$ or both $(n=1)$. Risk factors for a change in clinical management were previous neck surgery (OR 5.9, 95\% Cl: 1.4-24.5), surgery in a non-tertiary center (OR 13.4, 95\% Cl: 2.8 - 63.8), TSH <53.4 mU/L (OR 19.64, 95\% Cl: 4.94-78.13), thyroglobulin $\geq 50.0 \mathrm{ng} / \mathrm{L}(\mathrm{OR} 7.4,95 \% \mathrm{Cl}: 1.6-34.9)$ and free $\mathrm{T} 4 \geq 4.75 \mathrm{pmol} /$ L (OR 156.8, 95\% Cl: 128.4-864.2)

Conclusion: The Dx scan can potentially change clinical management before treatment with I-131, but the yield is low. A Dx-scan should only be considered for patients with a high pre-scan risk of a change in management, based on patient history and prior centerbased surgical outcomes.

Keywords: postoperative I-131 diagnostic scan, differentiated thyroid carcinoma, uptake, clinical management, thyroid cancer 


\section{INTRODUCTION}

For many years, the standard treatment for differentiated thyroid carcinoma (DTC) consisted of thyroidectomy, radio-active iodine (RAI) remnant ablation and/or (adjuvant) therapy, and TSH suppression therapy $(1,2)$. Nowadays, there is a trend towards de-escalation of treatment for patients with DTC, illustrated by new protocols with decreased I-131 activity for low- and intermediate risk patients $(1,3)$.

Since the implementation of recent guidelines, the role of the I-131 diagnostic scan (Dx scan) is being questioned. The Dx scan is used to detect a thyroid remnants or unknown lymph node metastases, potentially resulting either in pre-RAI additional surgery or a change in the administered I-131 activity (4). Furthermore, a local increased uptake at the original thyroid bed reflects the iodine uptake capacity of the thyroid remnant, which may result in an inflammatory reaction after I-131 therapy. The incidence and severity of this radiation-induced inflammation at the original thyroid bed is directly related to the uptake. This inflammation can be avoided by lowering the administered I-131 activity or by preventive prescription of anti-inflammatory drugs such as NSAIDs or corticosteroids (5). Previous literature reported additional findings on the Dx scan like unknown metastases or a remnant in $25-53 \%$ of DTC patients (6-8). However, whether these findings of the Dx scan actually changes clinical management remains unclear. Ideally, a Dx-scan should not be necessary when the quality of pre-operative staging and surgery itself is high, minimalizing the risk of a thyroid remnant or local metastases (9).

By studying the Dx scan in a specialized tertiary hospital including referrals from low-volume centers, we aim to examine the changes in clinical management based on the results of the Dx scan and to specify in which patients, if any, a Dx scan has additional value.

\section{MATERIAL AND METHODS}

\section{Study Population}

In this retrospective study, all DTC patients treated with I-131 from January 2005 until July 2015 at the University Medical Centre Groningen (UMCG) were included. Patients were excluded when a Dx scan was not performed or when recombinant human TSH (rhTSH) was used before RAI therapy. According to Dutch law, i.e. Medical Research Involving Human Subjects (WMO), no ethical review was necessary for retrospective data collection.

\section{Diagnostic and Treatment Protocol}

In patients with a palpable thyroid nodule, ultrasonography guided fine needle aspiration cytology was performed, either at the UMCG or at the referring non-tertiary hospital. At the same session, ultrasound characteristics of the thyroid nodule were described and screening for metastatic lymph nodes in the neck region took place. All patients included in this study underwent a thyroidectomy with lymph node dissection if indicated, either at the UMCG or at one of the 19 referring hospitals. The seventh edition of the AJCC cancer staging system was used (10). Patients were classified as low risk (age 20-45 years, minimally invasive follicular carcinoma or classic papillary carcinoma, T1-2N0M0, without thyroglobulin-antibodies) or high risk (all other patients), according to the Dutch risk stratification at that time (2). In addition, patients were retrospectively classified according to a simplified ATA risk stratification as low risk (Tx-T2, Nx-N0 and Mx-M0), intermediate risk (any T3 or N1 tumor) or high risk (any T4 or M1 tumor) (1). The hospitals where the surgery was performed were classified as tertiary- (high-volume specialized hospitals) vs. non-tertiary hospitals (low-volume non-specialized hospitals). In addition, information about the surgical procedure was obtained to assess whether the thyroidectomy was performed in one or two sessions ('onestep' versus 'two-step' procedure).

After thyroidectomy, patients did not start thyroid hormone substitution and they followed a low-iodine diet starting one week prior to the RAI therapy that was generally applied 4-5 weeks after thyroidectomy. Repeat ultrasonography was not performed before the first RAI therapy. High risk patients received $5550 \mathrm{MBq} \mathrm{I}-131$ and low risk patients received 3700 MBq I-131, according to the, at that time applicable, Dutch risk stratification criteria (11). RAI therapy was administered at discretion of the treating physician, but generally when the Dx scan at $24 \mathrm{~h}$ showed no previously unknown metastases and an uptake $<10 \%$. In cases with undetectable $\mathrm{Tg}$ and no uptake on the Dx scan, RAI therapy was still administered. TSH suppression therapy was started after RAI therapy to minimize potential tumor growth (12). Post-therapy planar whole-body scans were obtained at 7 days (from 2010 onwards, and combined with a SPECT/CT of the neck) or 10 days (before 2010) after administration of RAI therapy. Six months after RAI therapy, a $74 \mathrm{MBq}$ I- 131 whole body scan after $72 \mathrm{~h}$ was performed, together with measurement of a TSH-stimulated serum thyroglobulin for re-stratification and to evaluate the I131 therapy success.

Free T4 and TSH were measured at the time of the Dx scan, using immunochemiluminometric E-module assay (Roche Diagnostics, Indianapolis, IN, USA) with normal values of respectively $11.0-19.5 \mathrm{pmol} / \mathrm{L}$ and $0.5-4.0 \mathrm{mU} / \mathrm{l}$. For the measurement of thyroglobulin $(\mathrm{Tg})$, the $\mathrm{Tg}$ immunoradiometric assay (Tg-IRMA) by Thermo Fisher Scientific (Henningsdorf, Germany) was used, with a limit of detection of $0.1 \mathrm{ng} / \mathrm{mL}$ and a functional sensitivity of $0.3 \mathrm{ng} / \mathrm{mL}$.

\section{Dx Imaging Protocol}

Planar whole-body scans were performed 24 hours following the administration of $37 \mathrm{MBq}$ I-131. Until 2010 a dual-headed Siemens Multispect-2 camera was used with 3 static images (head/neck, thorax/abdomen, pelvic/upper legs) of $10 \mathrm{~min}$ each, using a high-energy collimator and a 15\% energy window centered at $364 \mathrm{KeV}$. From 2010 to 2015 imaging was performed on a Siemens Symbia S or T2 dual-headed gamma camera also with a high-energy collimator and the same centering of the energy window. Whole body planar images were recorded with a scan rate of $8 \mathrm{~cm} / \mathrm{min}$. The uptake at the original thyroid bed was measured by an external gamma-probe 
for 2 minutes (Isomed 2400, Nuklear Medizintecknik, Dresden, $\mathrm{GmbH})$. After subtracting a 2-minute background measurement at the upper leg, the uptake was presented as percentage of the administered activity of $37 \mathrm{MBq}$ I-131.

NSAIDs or corticosteroids were generally prescribed to patients with an uptake $>5 \%$ at the discretion of the treating physician to avoid radiation-induced inflammation and oedema in the neck. In patients with an uptake $>10 \%$, multidisciplinary consultation was arranged immediately with the surgeon, endocrinologist, nuclear physician and radiologist to either perform additional surgery after diagnostic imaging (such as a neck ultrasound, a I-131 SPECT/CT scan, or a head and neck MRI scan) or to decrease the standard activity dose of I-131.

\section{Impact of Dx Imaging on Patient Management}

To specify cases in which a Dx scan could be of value, we reported the actual changes in clinical management based on the outcomes of the Dx scan. A change in clinical management was defined as additional surgery or a change in the administered activity of I-131. The patient group who had changes in clinical management were compared with those without changes in management, by using logistic regressions. Minor changes like prescription of antiinflammatory drugs were also described. Radiation-induced inflammation was defined as physical complaints in the neck region occurring after the administration of $37 \mathrm{MBq}$ I-131 until 2 weeks after I-131 therapy. Information on prescription of drugs and physical complaints after I-131 therapy was extracted from the electronic patient files.

\section{Statistics}

All data were presented as number (percentage) for categorical variables, median (inter quartile range, IQR) for skewed variables, and as mean ( \pm standard deviation [SD]) for variables with a normal distribution. Odds ratios (OR) and 95\% Confidence Intervals (CI) were calculated for each variable. The biomarkers TSH, free T4 and Tg were dichotomized. Cut-off values were obtained by maximizing the Youden-index with a receiver-operating characteristic (ROC) curve. Binary logistic regression models were used, in which variables with a univariable significance of $\mathrm{p}<0.15$ were added to a multivariable model. Each variable that did not contribute with a significance of $\mathrm{p}<0.10$ was incrementally removed from the multivariable model. The fit of the model was reported by Nagelkerke's $R_{N}^{2}$. A p-value $<0.05$ was considered statistically significant. Missing data were excluded from analysis. All statistical analyses were performed using the IBM Statistical Package for Social Science (SPSS version 23).

\section{RESULTS}

In total 280 patients underwent a total thyroidectomy in our region followed by treatment with I-131 at the UMC Groningen from 2005 to 2015. Two patients were excluded as they received I-131 therapy with recombinant TSH and in 16 patients the data of the Dx scan was missing, leaving 262 patients available for analysis (Figure 1). Baseline characteristics are shown in Table $\mathbf{1 .}$
Patients had an average age of $48.0 \pm 17.7$ years and $69 \%$ of patients were female. The median uptake in the thyroid bed was $2.4 \%$ (interquartile range 1.3-4.5\%). The Dx scan showed additional findings in 99 patients $(26.3 \%)$. The uptake was > $5 \%$ in 52 of 262 patients (19.8\%) of which the uptake was $>10 \%$ in 15 patients $(5.7 \%)$. A total of 17 out of 262 patients $(6.5 \%)$ had no visible uptake in the original thyroid bed at the Dx scan and their uptake measurements varied between 0.1 and $2.3 \%$. The Dx scan showed unexpected regional and distant metastases in 18 patients (7.3\%). These 18 patients were already classified as highrisk according to the Dutch risk-stratification and the Dx scan did not alter the risk classification or administered I-131 activity. When using the simplified ATA-stratification, 6 of these 18 patients $(33.3 \%)$ where classified as high risk and $12(66.7 \%)$ as intermediate risk and the Dx scan also did not alter the ATA risk-stratification.

\section{Change in Patient Management}

In 11 patients $(4.2 \%)$ the Dx scan changed clinical management, of which 4 patients required additional surgery (uptake ranging from 16 to 27\%). The indication for additional surgery was confirmed with MRI of the neck region. A fifth patient with uptake of $42.9 \%$, first underwent re-surgery. Thereafter, the uptake was still $23.9 \%$ resulting in the administration of a decreased activity of I-131. In these 5 patients, pathological examination after resurgery revealed benign thyroid tissue in 3 patients and in 2 patients DTC in a thyroid remnant with additional lymph node metastases was found. For 6 patients with an uptake between 5.6 and $24.0 \%$ the I-131 activity was reduced by $50 \%$ due to high uptake on the Dx scan. A further 6 patients $(2.3 \%$ ) had an uptake of $>10 \%$ (range: $11.4-15.4 \%$ ) but required no additional surgery or lower activity of I-131 at the discretion of the treating physician.

Patients with a change in clinical management, more often had a history of previous neck surgery compared to patients without a change in clinical management (Table 2: OR 5.9, 95\% CI: 1.4-24.5). Of the 72 patients who underwent surgery in a non-tertiary hospital, 9 patients $(12.5 \%)$ had a change in clinical management compared to 2 patients $(0.1 \%)$ with first surgery in a tertiary hospital (OR 13.4, 95\% CI: 2.8 - 63.8). TNM-stage, age, gender and ATA risk classification were not predictors of change of management after the Dx-scan. In a univariate model the biomarkers TSH <53.4 mU/L (OR 19.64, 95\% CI: 4.94-78.13), thyroglobulin $\geq 50.0 \mathrm{ng} / \mathrm{L}$ (OR 7.4, 95\% CI: 1.6-34.9) and free $\mathrm{T} 4 \geq 4.75 \mathrm{pmol} / \mathrm{L}$ (OR 156.8, 95\% CI: 28.4-864.2) were all predictive of a change in clinical management. The regression model showed that free T4 $>4.75 \mathrm{pmol} / \mathrm{L}$ alone is the strongest risk factor for a change in clinical management $\left(R_{N}^{2} 0.549\right)$.

Furthermore, there was no association between uptake measurement and results of re-stratification with a diagnostic I-131 whole body scan 6 months after I-131 therapy (OR 1.0, 95\% CI: 0.9-1.1, p 0.891).

\section{Radiation-Induced Local Side Effects}

The uptake was $>5 \%$ in a total of 52 patients of which 21 patients (40.4\%) were prescribed anti-inflammatory drugs, without a decision for further intervention. A total of thirteen patients 


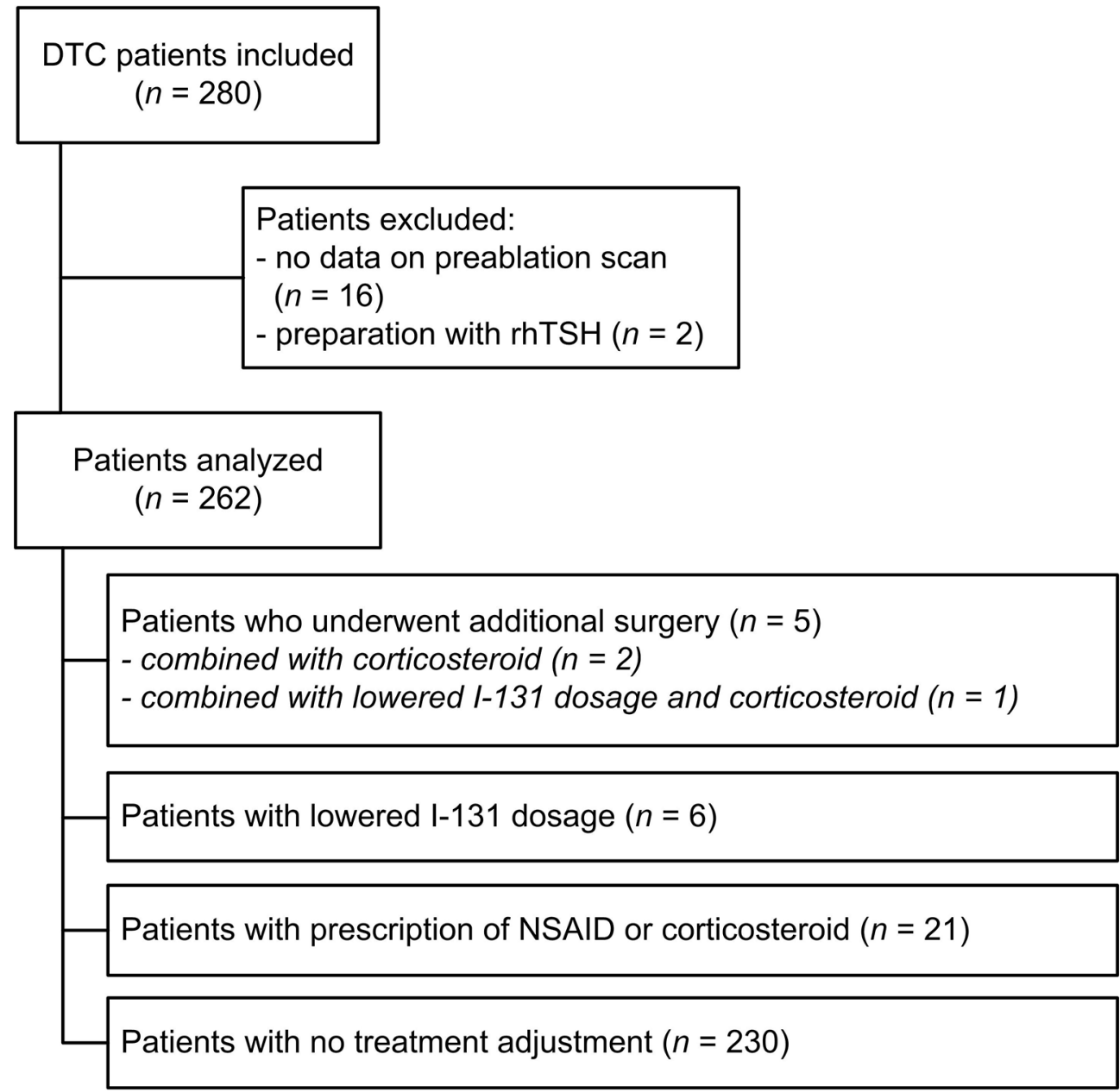

FIGURE 1 | Flowchart of inclusion and clinical management. DTC, differentiated thyroid carcinoma; NSAID, non-steroidal anti-inflammatory drugs; rhTSH, recombinant human thyrotropin.

(25.0\%) with an uptake of $>5 \%$ reported physical complaints during I-131 therapy. Five patients reported physical complaints during I-131 therapy despite using either NSAID's or corticosteroids. A further 7 patients reported physical complaints without being treated with anti-inflammatory drugs. One patient reported physical complaints despite reduction of I-131 activity. The reported complaints consisted of pain $(n=5)$ and swelling $(n=3)$ at the original thyroid bed, and inflammation of the throat $(n=5)$.

\section{DISCUSSION}

The Dx scan is used prior to I-131 treatment to select patients for additional surgery, lower I-131 activity, or to consider prescribing anti-inflammatory drugs prior to I-131 treatment. This current analysis of 262 Dx scans shows that $26.3 \%$ of Dx scans revealed additional findings, like no uptake, high uptake $(>5 \%)$, or unexpected regional or distant metastases. Other series have reported similar rates of unexpected findings ranging from $53 \%$ in a theoretical approach in 355 patients (6) to around $25 \%$ in other studies $(7,8)$. This would result in a 'number needed to scan' between approximately 2 to 4 , which would certainly justify the regular use of a Dx scan. But while our current study confirmed a high incidence of additional findings, a change in clinical management was only seen in $4.2 \%$ of patients. Retrospectively, the Dx scan did not alter the patient's risk class according to the Dutch risk classification and all of the patients with suggestion of regional lymph node or distant metastases on the Dx scan were ATA intermediate or high risk. In our study the Dx scan would therefore not have influenced the level of I-131 activity to be administered. Chen and colleagues also reported additional information in $25 \%$ of patients who underwent a Dx scan, but a change in clinical management only occurred in $5.7 \%$ of patients by changing the administration of I-131 in a two-step approach (8).

Besides limited clinical consequences, there are several other potential disadvantages linked to the Dx scan, including low 
TABLE 1 | Patient characteristics at DTC diagnosis.

\begin{tabular}{|c|c|}
\hline & All patients $(n=262)$ \\
\hline Mean age (years) \pm SD & $48.0 \pm 17.7$ \\
\hline \multicolumn{2}{|l|}{$\operatorname{Sex} n(\%)$} \\
\hline Male & $81(30.9)$ \\
\hline Female & $181(69.1)$ \\
\hline \multicolumn{2}{|l|}{ Histology n (\%) } \\
\hline Papillary & 206 (78.6) \\
\hline Follicular & 35 (13.3) \\
\hline Hürthle & $21(8.0)$ \\
\hline \multicolumn{2}{|l|}{ Procedure of surgery n (\%) } \\
\hline One-step & $141(53.8)$ \\
\hline Two-step & $121(46.2)$ \\
\hline \multicolumn{2}{|l|}{ Location of surgery n (\%) } \\
\hline Tertiary & $160(72.5)$ \\
\hline Non-tertiary & $72(27.5)$ \\
\hline \multicolumn{2}{|l|}{ TNM Tumor stage ${ }^{1} \mathrm{n}(\%)$} \\
\hline Tx-T2 & $142(54.2)$ \\
\hline T3-T4 & $120(45.8)$ \\
\hline $\mathrm{Nx}-\mathrm{NO}$ & $145(55.3)$ \\
\hline N1 & $117(44.7)$ \\
\hline $\mathrm{Mx}-\mathrm{MO}$ & $240(91.6)$ \\
\hline M1 & $22(8.4)$ \\
\hline \multicolumn{2}{|l|}{ Dutch risk stratification n (\%) } \\
\hline Low risk & $37(14.1)$ \\
\hline High risk & 225 (85.9) \\
\hline \multicolumn{2}{|l|}{ ATA risk stratification² n (\%) } \\
\hline Low risk & 87 (33.2) \\
\hline Intermediate risk & $143(54.6)$ \\
\hline High risk & $32(12.2)$ \\
\hline \multicolumn{2}{|l|}{ AJCC Cancer Stage 1 n (\%) } \\
\hline । & $131(50.0)$ \\
\hline$\|$ & $30(11.5)$ \\
\hline III & $34(13.0)$ \\
\hline IV & $67(25.6)$ \\
\hline Median TSH (mU/L) (IQR) & $96.0(66.25-131.0)$ \\
\hline
\end{tabular}

\section{${ }^{1}$ AJCC TNM 7th edition.}

${ }^{2}$ ATA risk stratification 2015

DTC, differentiated thyroid carcinoma; SD, standard deviation; TSH, thyrotropin; IQR, interquartile range; ATA, American Thyroid Association.

TABLE 2 | Patient and treatment characteristics associated with a change in clinical management.

\begin{tabular}{|c|c|c|c|c|c|}
\hline & $\begin{array}{l}\text { Unchanged } \\
\text { management } \\
(n=251)\end{array}$ & $\begin{array}{l}\text { Change in } \\
\text { management } \\
(n=11)\end{array}$ & $\begin{array}{l}\text { Odds } \\
\text { ratio }\end{array}$ & $95 \% \mathrm{Cl}$ & \\
\hline $\begin{array}{l}\text { Age (10 years) } \\
\text { mean } \pm \text { SD } \\
\text { Sex } n(\%)\end{array}$ & $48.0 \pm 18.0$ & $47.0 \pm 11.3$ & 0.97 & $\begin{array}{c}0.68- \\
1.36\end{array}$ & $p=0.85$ \\
\hline Male & 78 (31.1) & 3 (27.3) & Ref. & & \\
\hline Female & 173 (68.9) & $8(72.7)$ & 1.20 & $\begin{array}{c}0.31- \\
4.65\end{array}$ & $p=0.79$ \\
\hline \multicolumn{6}{|l|}{ Histology n (\%) } \\
\hline Papillary & 197 (78.5) & $9(81.8)$ & Ref. & & \\
\hline Follicular & $54(21.5)$ & 2 (18.2) & 0.81 & $\begin{array}{c}0.17- \\
3.86\end{array}$ & $p=0.79$ \\
\hline \multicolumn{6}{|l|}{$\begin{array}{l}\text { TNM Tumor } \\
\text { stage n (\%) } \\
\text { T stage }\end{array}$} \\
\hline Tx-T2 & 133 (53.0) & 9 (81.8) & Ref. & & \\
\hline T3-T4 & $118(47.0)$ & 2 (18.2) & 0.25 & $\begin{array}{c}0.05- \\
1.18\end{array}$ & $p=0.08$ \\
\hline
\end{tabular}

(Continued)
TABLE 2 | Continued

\begin{tabular}{|c|c|c|c|c|c|}
\hline & $\begin{array}{l}\text { Unchanged } \\
\text { management } \\
(n=251)\end{array}$ & $\begin{array}{l}\text { Change in } \\
\text { management } \\
(n=11)\end{array}$ & $\begin{array}{l}\text { Odds } \\
\text { ratio }\end{array}$ & $95 \% \mathrm{Cl}$ & \\
\hline \multicolumn{6}{|l|}{ N stage } \\
\hline $\mathrm{Nx}-\mathrm{NO}$ & $137(54.6)$ & 8 (72.7) & Ref. & & \\
\hline N1 & $114(45.4)$ & 3 (27.3) & 0.53 & $\begin{array}{c}0.28- \\
1.01\end{array}$ & $p=0.25$ \\
\hline \multicolumn{6}{|l|}{ M stage } \\
\hline $\mathrm{Mx}-\mathrm{MO}$ & 229 (91.2) & $11(100)$ & * & & \\
\hline M1 & $22(8.8)$ & 0 & & & \\
\hline \multicolumn{6}{|l|}{$\begin{array}{l}\text { ATA risk } \\
\text { classification } \\
\mathrm{n}(\%)\end{array}$} \\
\hline Low risk & 80 (31.9) & 7 (63.6) & Ref. & & \\
\hline \multicolumn{6}{|l|}{$\begin{array}{l}\text { History of neck } \\
\text { surgery n (\%) }\end{array}$} \\
\hline No & 235 (94.0) & 8 (72.7) & Ref. & & \\
\hline Yes & $15(6.0)$ & 3 (27.3) & 5.88 & $\begin{array}{l}1.41- \\
24.45\end{array}$ & $p=0.02$ \\
\hline \multicolumn{6}{|l|}{$\begin{array}{l}\text { Procedure of } \\
\text { surgery n (\%) }\end{array}$} \\
\hline One-step & 137 (54.6) & $4(36.4)$ & Ref. & & \\
\hline Two-step & $114(45.5)$ & 7 (63.6) & 2.10 & $\begin{array}{l}0.60- \\
7.67\end{array}$ & $p=0.24$ \\
\hline \multicolumn{6}{|l|}{$\begin{array}{l}\text { Location of first } \\
\text { surgery } n(\%)\end{array}$} \\
\hline Tertiary & 188 (74.9) & 2 (18.2) & Ref. & & \\
\hline Non-tertiary & $63(25.1)$ & $9(81.8)$ & 13.43 & $\begin{array}{l}2.83- \\
63.81\end{array}$ & $p=0.001$ \\
\hline \multicolumn{6}{|l|}{ TSH n (\%) } \\
\hline$\geq 53.4 \mathrm{mU} / \mathrm{L}$ & 30 (12.0) & 8 (72.7) & Ref. & & \\
\hline$<53.4 \mathrm{mU} / \mathrm{L}$ & 221 (88.0) & $3(27.3)$ & 19.64 & $\begin{array}{l}4.94- \\
78.13\end{array}$ & $p<0.001$ \\
\hline \multicolumn{6}{|l|}{ Free T4 n (\%) } \\
\hline$<4.75 \mathrm{pmol} / \mathrm{L}$ & 244 (97.2) & 2 (18.2) & Ref. & & \\
\hline$\geq 4.75 \mathrm{pmol} / \mathrm{L}$ & $7(2.8)$ & $9(81.8)$ & 156.8 & $\begin{array}{l}28.4- \\
864.2\end{array}$ & $p<0.001$ \\
\hline \multicolumn{6}{|l|}{$\begin{array}{l}\text { Thyroglobulin } n \\
(\%)\end{array}$} \\
\hline$<50.0 \mathrm{ng} / \mathrm{L}$ & $156(62.2)$ & 2 (18.2) & Ref. & & \\
\hline$\geq 50.0 \mathrm{ng} / \mathrm{L}$ & $95(37.8)$ & $9(81.8)$ & 7.39 & $\begin{array}{l}1.56- \\
34.93\end{array}$ & $p=0.01$ \\
\hline
\end{tabular}

Odds ratios with 95\% Cl: and p-values were calculated with an univariable logistic regression. Follicular thyroid carcinoma included Hürtle cell carcinoma.

${ }^{*}$ Could not be assessed.

Cl, confidence interval; DTC, differentiated thyroid carcinoma; ATA, American Thyroid Association; Ref., reference category.

sensitivity compared to the post-Tx scan and the risk of stunning. Stunning is a radiobiological phenomenon defined as a temporary suppression of the iodine trapping function of the thyrocytes and thyroid cancer cells as a result of the radiation given off by the first, low dose of I-131. Stunning can be avoided by using I-123 or by lowering the activity of I-131 to $37 \mathrm{MBq}$ as in our current protocol (13).

Both the limited effect on clinical management and the potential disadvantages imply a selected use of the Dx scan. To assist in selecting patients for a Dx scan, we identified several pre-scan risk factors for a change in management. High plasma levels of $\mathrm{Tg}$ and free T4, and low levels of TSH were associated 
with a higher likelihood of a change in management. Moreover, patients who had undergone first surgery in a non-tertiary center or had a history of previous neck surgery had a higher likelihood of a change in management, suggesting a relation with surgical approach and/or quality. Our study included patients from various low- and high-volume surgical centers, allowing to compare the risk for a change in management, based on surgical centers. The uptake on the Dx scan has previously also been reported to be lower after one-step thyroidectomy (versus two-step) and after surgery by more experienced surgeons $(14,15)$. Thus, the value of the Dx scan is largely determined by the risk of a thyroid remnant and thus quality of surgery. This last interpretation cannot be confirmed with our data, because we did not register parameters on quality of surgery. The outcome of thyroid surgery, in terms of complications and length of hospital stay, is also better with a higher volume operated by a thyroid surgeon $(9,16-18)$. So, while in general the Dx scan has limited influence on clinical management, it could be considered based on history of the patient, previous surgical outcomes in a center, and potentially on levels of $\mathrm{Tg}$ and free $\mathrm{T} 4$.

A major limitation of this study is the non-protocolled retrospective design. Six patients with an uptake of $>10 \%$ did not have a change in clinical management at discretion of the treating physician. This could result in an underestimation of the effect of the Dx scan, but these small number of patients do not significantly influence the results of the study. Referral bias could also result in a higher rate of management change for patients referred from low-volume centers, but because only our center treats DTC patients in the region with I-131 this effect is also very limited.

Using the Dx scan to deescalate treatment by lowering or even withholding I-131 could again increase the value of the Dx scan. This cannot be studied with the design of our study but has been reported earlier. In a study by Avram and colleagues. the Dx scan was involved in individualizing I-131 activities (19). The Dx scan was used to stratify patients on risk of recurrence. Subsequently, dosimetry calculations were performed based on whole body uptake counts and blood activity counts. This approach resulted

\section{REFERENCES}

1. Haugen BR, Alexander EK, Bible KC, Doherty GM, Mandel SJ, Nikiforov YE, et al. 2015 American Thyroid Association Management Guidelines for Adult Patients With Thyroid Nodules and Differentiated Thyroid Cancer: The American Thyroid Association Guidelines Task Force on Thyroid Nodules and Differentiated Thyroid Cancer. Thyroid (2016) 26(1):1-133. doi: 10.1089/ thy. 2015.0020

2. Schildkliercarcinoom. (2015). Available at: https://www.oncoline.nl/ schildkliercarcinoom.

3. Kim BW, Yousman W, Wong WX, Cheng C, McAninch EA. Less is More: Comparing the 2015 and 2009 American Thyroid Association Guidelines for Thyroid Nodules and Cancer. Thyroid (2016) 26(6):759-64. doi: 10.1089/ thy.2016.0068

4. Avram AM, Esfandiari NH, Wong KK. Preablation 131-I Scans With SPECT/ CT Contribute to Thyroid Cancer Risk Stratification and 131-I Therapy Planning. J Clin Endocrinol Metab (2015) 100(5):1895-902. doi: 10.1210/ jc.2014-4043 in a complete response after a single RAI therapy in $88 \%$ of patients without distant metastases, opening a new potential approach to the Dx scan.

\section{CONCLUSION}

The Dx scan before RAI therapy in patients with differentiated thyroid cancer has a low yield regarding change of clinical management. Selective use of the Dx scan in patients with a medical history of previous neck surgery or surgically treated in a in low-volume hospital may have advantages. Whether to perform a Dx-scan should be considered based on patient characteristics and prior center-based surgical outcomes.

\section{DATA AVAILABILITY STATEMENT}

The original contributions presented in the study are included in the article/supplementary material. Further inquiries can be directed to the corresponding author.

\section{ETHICS STATEMENT}

Ethical review and approval was not required for the study on human participants in accordance with the local legislation and institutional requirements. Written informed consent for participation was not required for this study in accordance with the national legislation and the institutional requirements.

\section{AUTHOR CONTRIBUTIONS}

All authors contributed to the article and approved the submitted version. TB and $\mathrm{WZ}$ analyzed the data and wrote the manuscript with input from all authors.
5. Cherk MH, Kalff V, Yap KS, Bailey M, Topliss D, Kelly MJ. Incidence of Radiation Thyroiditis and Thyroid Remnant Ablation Success Rates Following $1110 \mathrm{MBq}(30 \mathrm{mCi})$ and $3700 \mathrm{MBq}(100 \mathrm{mCi})$ Post-Surgical 131I Ablation Therapy for Differentiated Thyroid Carcinoma. Clin Endocrinol (Oxf) (2008) 69(6):957-62. doi: 10.1111/j.1365-2265.2008.03276.x

6. Van Nostrand D, Aiken M, Atkins F, Moreau S, Garcia C, Acio E, et al. The Utility of Radioiodine Scans Prior to Iodine 131 Ablation in Patients With Well-Differentiated Thyroid Cancer. Thyroid (2009) 19(8):849-55. doi: 10.1089/thy.2008.0419

7. Avram AM, Fig LM, Frey KA, Gross MD, Wong KK. Preablation 131-I Scans With SPECT/CT in Postoperative Thyroid Cancer Patients: What is the Impact on Staging? J Clin Endocrinol Metab (2013) 98(3):1163-71. doi: 10.1210/jc.2012-3630

8. Chen MK, Yasrebi M, Samii J, Staib LH, Doddamane I, Cheng DW. The Utility of I-123 Pretherapy Scan in I-131 Radioiodine Therapy for Thyroid Cancer. Thyroid (2012) 22(3):304-9. doi: 10.1089/thy.2011.0203

9. Adam MA, Thomas S, Youngwirth L, Hyslop T, Reed SD, Scheri RP, et al. Is There a Minimum Number of Thyroidectomies a Surgeon Should Perform to Optimize Patient Outcomes? Ann Surg (2017) 265(2):402-7. doi: 10.1097/ SLA. 0000000000001688 
10. Edge S, Byrd DR, Compton CC, Fritz AG, Greene FL, Trotti A. Ajcc Cancer Staging Manual, 7th Edition. France: Springer (2010).

11. Schildkliercarcinoom. (2007). Landelijke richtlijn, versie 1.0 (Dutch Thyroid Cancer Guidelines).

12. Cooper DS, Specker B, Ho M, Sperling M, Ladenson PW, Ross DS, et al. Thyrotropin Suppression and Disease Progression in Patients With Differentiated Thyroid Cancer: Results From the National Thyroid Cancer Treatment Cooperative Registry. Thyroid (1998) 8(9):737-44. doi: 10.1089/thy.1998.8.737

13. Van Nostrand D. To Perform or Not to Perform Radioiodine Scans Prior to 131I Remnant Ablation? Pro. In: L Wartofsky, D Van Nostrand, editors. Thyroid Cancer. NY: Springer (2016).

14. Song JSA, Moolman N, Burrell S, Rajaraman M, Bullock MJ, Trites J, et al. Use of Radioiodine-131 Scan to Measure Influence of Surgical Discipline, Practice, and Volume on Residual Thyroid Tissue After Total Thyroidectomy for Differentiated Thyroid Carcinoma. Head Neck (2018) 40(10):2129-36. doi: 10.1002/hed.25204

15. Yap BK, Homer J, Parrott N, Loughran S, Murby B, Swindell R, et al. Use of PreAblation Radioiodine-131 Scan to Assess the Impact of Surgical Volume and Specialisation Following Thyroidectomy for Differentiated Thyroid Carcinoma. Eur Thyroid J (2013) 2(4):275-81. doi: 10.1159/000355911

16. Gourin CG, Tufano RP, Forastiere AA, Koch WM, Pawlik TM, Bristow RE. Volume-Based Trends in Thyroid Surgery. Arch Otolaryngol Head Neck Surg (2010) 136(12):1191-8. doi: 10.1001/archoto.2010.212
17. Sosa JA, Bowman HM, Tielsch JM, Powe NR, Gordon TA, Udelsman R. The Importance of Surgeon Experience for Clinical and Economic Outcomes From Thyroidectomy. Ann Surg (1998) 228(3):320-30. doi: 10.1097/ 00000658-199809000-00005

18. Stavrakis AI, Ituarte PH, Ko CY, Yeh MW. Surgeon Volume as a Predictor of Outcomes in Inpatient and Outpatient Endocrine Surgery. Surgery (2007) 142 (6):887-99; discussion -99. doi: 10.1016/j.surg.2007.09.003

19. Avram AM, Rosculet N, Esfandiari NH, Gauger PG, Miller BS, Cohen M, et al. Differentiated Thyroid Cancer Outcomes After Surgery and Activity-Adjusted 131i Theragnostics. Clin Nucl Med (2019) 44(1):11-20. doi: 10.1097/ RLU.0000000000002321

Conflict of Interest: The authors declare that the research was conducted in the absence of any commercial or financial relationships that could be construed as a potential conflict of interest.

Copyright () 2021 van der Boom, Zandee, Dekkers, van der Horst-Schrivers, Jansen, Kruijff, Brouwers and Links. This is an open-access article distributed under the terms of the Creative Commons Attribution License (CC BY). The use, distribution or reproduction in other forums is permitted, provided the original author(s) and the copyright owner(s) are credited and that the original publication in this journal is cited, in accordance with accepted academic practice. No use, distribution or reproduction is permitted which does not comply with these terms. 